\title{
CALCULATION OF THE CONDENSATE FRACTION IN LIQUID HELIUM-4
}

\author{
A. A. Rovenchak, I. O. Vakarchuk \\ Department for Theoretical Physics, \\ Ivan Franko National University of Lviv, \\ 12 Drahomanov St., Lviv, Ukraine, UA-79005
}

(Received December 25, 2006)

\begin{abstract}
We adduce the results of the condensate fraction calculation for liquid helium-4. The method is derived from the first principles and involves minimum assumptions. The only experimental quantity we need in our calculations is the static structure factor which is easily measurable. We use the approximation in which expressions contain one summation in the wave vector space (or one integration it the radius vector space) in order to demonstrate the validity of our method. The computed values of the condensate fraction lie within $8.8 \div 14 \%$ depending on the model potential for the short-range interaction. This result agrees with recent experimental measurements and numerical estimations.
\end{abstract}

Key words: liquid helium, Bose-condensate, condensate fraction.

PACS number(s): 67.10.Ba, 67.25.D-

\section{INTRODUCTION}

The phenomenon of Bose-Einstein condensation and superfluidity in Bose-systems is a subject of great interest. Superfluidity in liquid helium-4 can be easily observed now but the measurements of the condensate fraction remain a difficult problem because of the strong interactions in this fluid. Researchers, both experimentalists and theorists, now generally concur that $\sim 10 \%$ of atoms are in the lowest (zero momentum) state at zero temperature. The relative number of atoms with zero momentum is called condensate fraction, here we denote it as $f$.

The problem of the calculation of condensate fraction in liquid helium- 4 was for the first time considered in the classical paper by Penrose and Onsager [1]. The authors used a very rough approximation of hard spheres for the ground-state wavefunction and calculated this quantity as approximately 0.08 or $8 \%$.

During recent twenty years different methods for measurements and theoretical calculations of the condensate fractions were used. Neutron scattering is used to draw the information on the momentum distribution and hence on the condensate fraction. Sears et al. [2] obtained the value $13.9 \%$. Later, a similar value of $13.3 \%$ was obtained by Sears [3] using the data on temperature variations of the average kinetic energy.

Numerical studies based on the variational method were carried out in the series of works by Manousakis, Pandharipande et al. [4-6] by means of Jastrow wavefunction with three-body corrections. Their results vary within $8.2 \div 10.3 \%$.

The numerical estimations of the condensate fractions were also made in the series of works by Vakarchuk et al. and Vakarchuk [7-9] leading to the results of $3.7 \div 8.8 \%$. The methods used in these papers are based on the meanspherical approximation for the structure functions of liquid helium-4 at zero temperature [7], direct quantummechanical computations from the first principles $[8,9]$.
The value of approximately $10 \%$ was obtained for $f$ by Sokol and collaborators by means of deep-inelastic neutron scattering at high-momentum transfer [10-12]. The experimental result of $\simeq 6 \%$ was obtained by Azuah et al. [13] from high precision neutron scattering. High precision measurements of the dynamic structure factor allowed Glyde et al. [14] to derive the information on the condensate fraction at zero temperature as well as its temperature dependence. They report $f=7.25 \pm 0.75 \%$ at $0 \mathrm{~K}$.

Monte Carlo (MC) methods were also used to study the question of the condensate fraction. The result of Whitlock and Panoff [15] is $\simeq 9 \%$ (Green Function MC). Ceperley and Pollock [16] obtained the value $\simeq 7 \%$ using Path Integral MC technics at $1.18 \mathrm{~K}$. Also, Moroni et al. [17] give $7.2 \%$ from Diffusion MC simulations. The recent estimation from Quantum MC calculations by Moroni and Boninsegni [18] is $6.9 \pm 0.5 \%$.

A series of papers by Mayers et al. $[19,20]$ was devoted to both calculation of the condensate fraction based on phenomenological assumptions [19] (the result being $9.9 \%$ ) and measurements on the high-energy scattering [20] $(f=15 \pm 4 \%$ at $1.3 \mathrm{~K})$. A semi-phenomenological method for extracting the condensate fraction data was proposed also by Rinat and Taragin [21]. The authors obtained $f$ at different temperatures and extrapolated their results to $0 \mathrm{~K}$ as $f=9.0 \pm 0.3 \%$.

It is interesting to note that the results of recent neutron scattering experiments for helium-4 confined in $\mathrm{Vy}-$ cor [22] and helium films [23] are comparable with bulk values.

\section{METHOD OVERVIEW}

The aim of this work is to show a possibility of calculation of the condensate fraction without drawing any special huge computational efforts. 
The method we use was worked out in [9]. The condensate fraction is calculated directly from the singleparticle distribution function $F_{1}(R)$ as its long-range limit:

$$
f=N_{0} / N=\lim _{R \rightarrow \infty} F_{1}(R)
$$

where $N_{0}$ is the number of particles in zero momentum state and $N$ is the total number of particles in the system.

Our techniques do not involve any phenomenological assumptions. For the computations we use as input only one experimental quantity, the static structure factor of helium at zero temperature. It is an advantage in comparison with those methods for which such quantities as the dynamic structure factor is necessary. Its precise measurement is a much more complicated problem than that of the static structure factor.

The method is fully controlled, i. e., we always know which effects are neglected, and it is not difficult to take them into account in the next approximation (in principle). Here we present only the first approximation (RPA) but the expressions are easily extendable in order to take subtler effects like many-particle interactions.

The only significant problem arising while extending our method further is the knowledge of the static threeparticle structure factor. Unlike the usual structure factor, it is not an easily measurable quantity already. One of the ways for its calculation we see in the following scheme: expressions for the three-particle distribution function $F_{3}$ (beyond superposition approximation) containing pair distribution function $\rightarrow 6$-dimensional Fourier transformation of $F_{3}$ leading to the three-particle structure factor. A separate paper will be devoted to this problem.

We consider three different approaches for the shortrange repulsive interactions. In the first one, they are not taken it into account explicitly at all. In the second approach this repulsion is modeled by Meyer's function $e^{(A / R)^{n}}-1, R$ is the radius-vector absolute value. We chose $A=2.1 \AA$ and $n=12$. This potential is referred here as 'almost hard spheres' (AHS) [24]. The third model is the potential of hard spheres (HS) with the diameter $2.1 \AA$.

\section{CALCULATING PROCEDURE}

The principal calculating procedure consists of the following steps:

1. Input: Experimental data on the structure factor [25] (should be converted into zero temperature $[26])$;

2. Input: Model for the short-range interactions (HS, AHS or none) [24];

3. Output: Structure factor of the model system corresponding to the short-range repulsion;
4. Output: Fourier transformation of the interatomic potential (effective) [24,26];

5. Output: Pair distribution function $F_{2}(R)$ [25];

6. Result: Condensate fraction.

In this work, we propose a simple method for the calculation of the condensed fraction using the formulae obtained earlier in [8]:

$$
\begin{aligned}
& f=\exp \left(I_{1 A}+\Delta_{1} J+\ldots\right), \\
& I_{1 A}=-\frac{1}{4 N} \sum_{\mathbf{q} \neq 0}\left(\alpha_{q}-1\right)^{2} / \alpha_{q}, \\
& \Delta_{1} J=\varrho \int d \mathbf{R}\left[2 h^{*}(R)-h(R)+h^{2}(R) / 4\right], \\
& h(R)=F_{2}(R)-1, \quad h^{*}(R)=\sqrt{F_{2}(R)}-1,
\end{aligned}
$$

dots denote terms having more than one integration over wave vector or coordinate, $F_{2}(R)$ is the pair distribution function (PDF), $N$ is the number of atoms, and

$$
\alpha_{q}=\sqrt{1+2 \varrho \nu_{q} / \frac{\hbar^{2} q^{2}}{2 m}},
$$

$\varrho$ is helium density, $\varrho=0.02185 \AA^{-3}, m$ is the mass of helium atom, $m=4.0026$ a. m. u., $q$ is the wave vector.

The expression for PDF within the accepted approach was obtained in [8]:

$$
F_{2}(R)=\exp \left\{\frac{1}{N} \sum_{\mathbf{q} \neq 0} \frac{2 a_{2}(\mathbf{q})}{1-2 a_{2}(\mathbf{q})} e^{i \mathbf{q R}}+\ldots\right\},
$$

while the self-consistent description of short- and longrange correlations in liquid helium-4 leads to the following result for the function $a_{2}$ [27]:

$$
a_{2}(\mathbf{q})=\frac{1}{2}\left(\frac{1}{S_{q}^{\text {s.r. }}}-\frac{1}{S_{q}}\right)
$$

with $S_{q}^{\text {s.r. }}$ being the structure factor of the model system (it equals 1 if the short-range repulsion is not given explicitly), superscript 's. r.' corresponds to 'short-range'. $S_{q}$ is the experimental structure factor (at $0 \mathrm{~K}$ ). These expressions contain $\nu_{q}$ being the Fourier image of the interatomic potential in helium that has an effective nature. It is obtained from the first principles using the collective variables formalism in the Schrödinger equation, as described in [26].

At step (4) of the calculation procedure we encountered problems while considering the HS short-range potential. The reason is clear: a weak damping of the structure functions at large values of wave vector or radius vector requires much more careful numerical computations than those in two other cases.

Some usual problems appear at step (5) of the calculating procedure where we smoothed $F_{2}(R)$ calculated from (3) and truncated it at the distances $R<2.2 \AA$. We put $F_{2}(R<2.2 \AA)=0$ in order to obtain the correct 


\section{A. A. ROVENCHAK, I. O. VAKARCHUK}

PDF at small distances [28]. In Fig. 1, we present the calculated PDFs in comparison with the experimental one (being more precise, derived from the measurements on the liquid structure factor) [28].

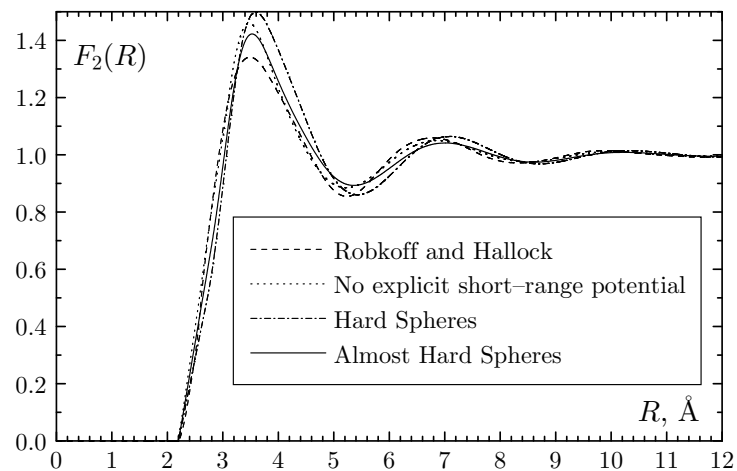

Fig. 1. Pair distribution function. The dashed line corresponds to the experimental data by Robkoff and Hallock [28] at $1.38 \mathrm{~K}$. The dotted line represents the smoothed data in the case when no explicit short-range repulsion is given. The dashed-dotted and solid lines are PDFs for HS and AHS short-range potentials, respectively.

\section{RESULTS AND DISCUSSION}

We have calculated the condensate fraction at zero temperature using different models for the short-range repulsive part of the potential.

The calculated values of $f$ are:

- No explicit short-range potential $f=14 \%$;

- AHS short-range potential $f=11 \%$;
- HS short-range potential $f=8.8 \%$.

We expect the real value being about $10 \div 30 \%$ less than the calculated one due to the contribution of the higher-order terms, as described in [8]. But in the first approximation we consider it to be a good accuracy for $f$ in liquid helium-4.

Our result for the condensate fraction is within the range of theoretical, numerical and experimental estimations mentioned here in the introductory part of the paper. Since our expressions are derived from the first principles, one can use the number for $f$ calculated in this way as a test for the condensate fraction information indirectly extracted from the experimental measurements. This becomes possible after the next approximations are computed.

We found that the short-range repulsive part of the potential has a significant influence on the results for the condensate fraction. Namely, when the short-range interactions are included into consideration explicitly, the results appear to be essentially dependent on the "hardness" of the core. A proper model for this interaction will be found when more terms are taken into account in the expressions (1), (3). The reason is that in principle the summation of the whole series corresponds to the correct calculation of the short-range interactions [27]. On the other hand, the condensate fraction in liquid helium- 4 is a very sensitive (with respect to the order of approximation) quantity. Thus, we can expect that having found such a model we obtain a possibility to use simple RPAlike expressions instead of higher-order ones. By now, the AHS ( $n=12, A=2.1 \AA$ ) potential seems to be the most suitable for this purpose.
[1] O. Penrose, L. Onsager, Phys. Rev. 104, 576 (1956).

[2] V. F. Sears, E. C. Svensson, P. Martel, A. D. B. Woods, Phys. Rev. Lett. 49, 279 (1982).

[3] V. F. Sears, Phys. Rev. B 28, 5109 (1983).

[4] E. Manousakis, V. R. Pandharipande, Q. N. Usmani, Phys. Rev. B 31, 7022 (1985).

[5] E. Manousakis, V. R. Pandharipande, Phys. Rev. B 31, 7029 (1985).

[6] E. Manousakis, V. R. Pandharipande, Q. N. Usmani, Phys. Rev. B 43, 13587 (1991).

[7] I. A. Vakarchuk, V. M. Migal, Ukr. Fiz. Zh. 32, 786 (1987).

[8] I. A. Vakarchuk, Ukr. Fiz. Zh. 35, 1261 (1990).

[9] I. A. Vakarchuk, Theor. Math. Phys. 80, 983 (1989); 82, 308 (1990).

[10] T. R. Sosnick, W. M. Snow, P. E. Sokol, R. N. Silver, Europhys. Lett. 9, 707 (1989).

[11] T. R. Sosnick, W. M. Snow, P. E. Sokol, Phys. Rev. B 41, 707 (1990).

[12] T. R. Sosnick, W. M. Snow, R. N. Silver, P. E. Sokol, Phys. Rev. B 43, 216 (1991).

[13] R. T. Azuah, W. G. Stirling, H. R. Glyde, M. Boninsegni, P. E. Sokol, S. M. Bennington, Phys. Rev. B 56,
14620 (1997).

[14] H. R. Glyde, R. T. Azuah, W. G. Stirling, Phys. Rev. B 62, 14337 (2000).

[15] P. A. Whitlock, R. M. Panoff, Can. J. Phys. 65, 1409 (1987).

[16] D. M. Ceperley, E. L. Pollock, Can. J. Phys. 65, 1416 (1987).

[17] S. Moroni, G. Senatore, S. Fantoni, Phys. Rev. B 55, 1040 (1997).

[18] S. Moroni, M. Boninsegni, J. Low Temp. Phys. 136, 129 (2004).

[19] J. Mayers, Rutherford Appleton Laboratory Technical Report RAL-TR-96-031 (1996).

[20] J. Mayers, C. Andreani, D. Colognesi, J. Phys.: Cond. Matt. 9, 10639 (1997).

[21] A. S. Rinat, M. F. Taragin, J. Low. Temp. Phys. 123, 139 (2001).

[22] R. T. Azuah, H. R. Glyde, R. Scherm, N. Mulders, B. Fåk, J. Low Temp. Phys. 130, 557 (2003).

[23] J. V. Pearce, S. O. Diallo, H. R. Glyde, R. T. Azuah, T. Arnold, J. Z. Larese, J. Phys.: Cond. Matt. 16, 4391 (2004).

[24] I. O. Vakarchuk, A. A. Rovenchak, J. Phys. Stud. 5, 126 
(2001); see also cond-mat/0104008 (2001).

[25] E. C. Svensson, V. F. Sears, A. D. B. Woods, P. Martel, Phys. Rev. B 21, 8 (1980).

[26] I. O. Vakarchuk, V. V. Babin, A. A. Rovenchak, J. Phys. Stud. 4, 16 (2000).
[27] I. A. Vakarchuk, I. R. Yukhnovskii, Theor. Math. Phys. 40, 626 (1979).

[28] H. N. Robkoff, R. B. Hallock, Phys. Rev. B 24, 159 (1981).

\title{
РОЗРАХУНОК КОНДЕНСАТНОЇ ФРАКЦІЇ В РІДКОМУ ГЕЛІЇ-4
}

\author{
А. А. Ровенчак, I. О. Вакарчук \\ Кафедра теоретичної фізики, \\ Львівсъкий націоналъний університет імені Івана Франка, \\ вул. Драгоманова, 12, Лъвів, Україна, 79005
}

\begin{abstract}
У статті наведено результати обчислення конденсатної фракції для рідкого гелію-4. Метод грунтується на перших принципах із залученням мінімальної кількости припущень. Єдиною експериментальною величиною, необхідною для обчислень, є статичний структурний фактор, який легко виміряти. Для демонстрації обгрунтованости методу використано наближення, в якому вирази містять одну суму за хвильовим вектором (або один інтеграл в $\mathbf{R}$-просторі). Обчислені значення конденсатної фракції лежать у межах $8.8 \div 14 \%$ залежно від модельного потенціялу короткосяжних взаємодій. Цей результат узгоджується зі сучасними експериментальними вимірюваннями й числовими оцінками.
\end{abstract}

